\title{
Surgical and post-operative treatment of chronic thromboembolic pulmonary
}

\section{hypertension}

\author{
E. Mayer
}

ABSTRACT: Patients with chronic thromboembolic pulmonary hypertension (CTEPH) have fibrous obstructions in their proximal pulmonary arteries, as well as a variable degree of secondary vascular remodelling of distal, surgically inaccessible, small pulmonary vessels.

Pulmonary endarterectomy (PEA) is currently the only potentially curative treatment option for CTEPH, although it is not known whether it has a beneficial effect on established secondary remodelling. Medical pre-treatment with therapies specific for pulmonary hypertension is often used as a bridge to PEA.

The aim of PEA is the mechanical removal of the proximal fibrous pulmonary artery obstruction as completely as possible while avoiding vascular injury within the lung. The challenge is to find and follow the correct pulmonary artery endarterectomy plane in the time allowed during brief periods of circulatory arrest.

Learning the operative technique is a complex process and, thus, early mortality after PEA decreases with increasing levels of surgical training and experience. Most patients experience lasting symptomatic and haemodynamic improvements after PEA, with only 10-20\% having persistent or recurrent pulmonary hypertension after surgery.

KEYWORDS: Chronic thromboembolic pulmonary hypertension, haemodynamics, pulmonary endarterectomy, pulmonary hypertension, pulmonary vascular resistance

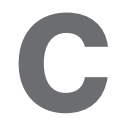

hronic thromboembolic pulmonary hypertension (CTEPH) is defined as symptomatic pulmonary hypertension (mean pulmonary artery pressure $(P \mathrm{pa})>25 \mathrm{mmHg})$ with persistent lung perfusion defects [1]. Patients with CTEPH have fibrous obstructions in their proximal pulmonary arteries, and this mechanical component of the disease is judged to be amenable to surgery. CTEPH can be worsened by a variable degree of secondary vascular remodelling of distal, surgically inaccessible, small pulmonary vessels, which can occur in regions served by open proximal vessels and regions downstream of the occluded vessels [2]. This review provides an understanding of the surgical treatment, as well as the pre-operative and post-operative management of patients with CTEPH.

\section{SURGICAL MANAGEMENT}

\section{Indication for surgery}

Pulmonary endarterectomy (PEA) is currently the only potentially curative treatment option for patients with CTEPH. However, it is not known whether PEA has an effect on the risk of subsequent arteriopathy. It is imperative that a diagnosis of CTEPH has been established before surgery is considered, and patients must have undergone anticoagulation therapy for at least 3 months. Patients being considered for surgery should be evaluated by an interdisciplinary team of medical and surgical specialists experienced in PEA using high-quality imaging technologies [3]. The majority of patients selected for surgery are in New York Heart Association (NYHA) functional class III or $\mathrm{IV}$, having dyspnoea at low levels of exertion or at rest. Surgery may also be considered in patients in NYHA functional class II (i.e. with exertional dyspnoea) and with close to normal pulmonary vascular resistance (PVR) at rest, if PVR increases significantly with exertion. In these patients, PEA will improve the ventilation-perfusion balance. Treatment of the disease at a relatively mild stage may, in time, help to minimise the development of secondary pulmonary arteriopathy [4].
CORRESPONDENCE

E. Mayer

Kerckhoff Clinic Heart and Lung Centre

Benekestr. 2-8

61231 Bad Nauheim

Germany

E-mail: e.mayer@kerckhoff-klinik.de

Received:

Nov 232009

Accepted after revision:

Dec 222009

PROVENANCE

Publication of this peer-reviewed article was supported by Bayer Schering Pharma AG, Germany (principal sponsor, European Respiratory Review issue 115).
European Respiratory Review Print ISSN 0905-9180 Online ISSN 1600-0617 
Mean PVR in patients undergoing PEA is $800-1,000 \mathrm{dyn} \cdot \mathrm{s} \cdot \mathrm{cm}^{-5}$ [5]. PVR is an important predictor of peri-operative mortality and, as such, a good prognostic marker for surgery. A pulmonary hypertension database study from the UK that included $>450$ patients with CTEPH showed a five-fold higher post-PEA mortality in the group of patients with a preoperative PVR of $>1,200$ dyn $\cdot \mathrm{s}^{\cdot} \mathrm{cm}^{-5}$ than in those with a pre-operative PVR of $<900 \mathrm{dyn} \cdot \mathrm{s} \cdot \mathrm{cm}^{-5}$ [6].

\section{Evaluation of operability}

CTEPH is considered inoperable in $20-40 \%$ of cases, with the proportion of patients deemed inoperable differing between countries and specialist centres, and depending on patient referral patterns, the specialty of the clinical department where the patient is assessed and the surgical experience of the operating team $[4,7,8]$. It is vital to perform pre-operative risk stratification in patients with CTEPH who are being considered for PEA. Reasons for a decision of inoperability include comorbidity (e.g. significant chronic obstructive pulmonary disease or severe left ventricular dysfunction), as well as the presence of certain risk factors, such as microvascular disease or a PVR of $>1,200 \mathrm{dyn} \cdot \mathrm{s} \cdot \mathrm{cm}^{-5}[7,9-11]$.

\section{Medical pre-treatment}

Medical pre-treatment with specific pulmonary hypertension therapies is often used as a bridge to PEA $[12,13]$, particularly in Europe; although efficacy data from randomised clinical trials are not available. In the UK, $65 \%$ of patients with CTEPH who underwent a PEA at a pulmonary hypertension centre between 2003 and 2006 received pre-treatment with prostanoids, an endothelin receptor antagonist or a phosphodiesterase- 5 inhibitor [8]. Only $\sim 18 \%$ of patients with CTEPH who were referred for treatment at specialist centres in Germany and Switzerland between 2008 and 2009 were not pre-treated with a drug specific for pulmonary hypertension. The remaining $82 \%$ received either monotherapy or combination therapy with pulmonary hypertension specific medications, including prostanoids, endothelin receptor antagonists and phosphodiesterase-5 inhibitors (table 1).

\section{Surgical technique}

The aim of surgery is the mechanical removal of the proximal fibrous pulmonary artery obstruction as completely as possible while avoiding vascular injury within the lung, which might

\section{TABLE 1 Medical pre-treatment with therapies specific for pulmonary hypertension $(\mathrm{PH})$ as a bridge to pulmonary endarterectomy}

\begin{tabular}{lc} 
Medical therapy for PH & Patients \\
\hline PH specific monotherapy & $35(70)$ \\
PH specific combination therapy & $6(12)$ \\
No PH specific therapy & $9(18)$ \\
\hline & \\
Data are presented as n (\%). * : data obtained following personal experience at \\
the Giessen University Hospital (Giessen, Germany), the Kerkhoff Clinic (Bad \\
Nauheim, Germany) and Zürich and Bern University Hospitals (both \\
Switzerland) between January 2008 and March 2009.
\end{tabular}

cause a fatal complication. During the course of CTEPH, distal, small pulmonary vessels can undergo vascular remodelling, but it is not known whether PEA has a beneficial effect on remodelling that is established at the time of surgery.

The surgical technique for PEA was developed and standardised at the University of California (San Diego, CA, USA) [15], although protocols vary even among expert centres. PEA requires bilateral proximal pulmonary artery incisions, and endarterectomy is carried out gently using small, blunt instruments (fig. 1). The procedure involves the establishment of extracorporeal circulation under conditions of deep hypothermia to $20^{\circ} \mathrm{C}$ and is carried out during brief periods of circulatory arrest, which are limited to $20 \mathrm{~min}[6,15,16]$. The challenge of PEA is to find and follow the correct pulmonary artery endarterectomy plane in the time allowed during circulatory arrest. The operative technique is complex and requires substantial training and experience.

\section{POST-OPERATIVE MANAGEMENT}

Haemodynamics immediately following surgery are usually very different from those prior to surgery, with patients falling into one of three categories. 1$)$ Most ( $\sim 70 \%)$ patients will have an abrupt, significant decrease in $P$ pa together with an increased cardiac output and systemic vasodilation. There is no need for intense medical therapy in these patients and management includes cautious fluid administration, reduction of cardiac output to avoid pulmonary overflow and administration of vasoconstrictors. 2) In a fifth of patients, $P$ pa will remain elevated, together with an increased cardiac output and systemic vasodilation. These patients are at risk of pulmonary reperfusion oedema and secondary right ventricular failure, and will require cautious fluid therapy, cardiac output reduction and diuretics. 3) Finally, in $\sim 10 \%$ of patients, $P$ pa will remain highly elevated, together with a low cardiac output and right ventricular and multi-organ failure. These patients are at severe risk of further haemodynamic deterioration and death. Management includes fluid restriction,

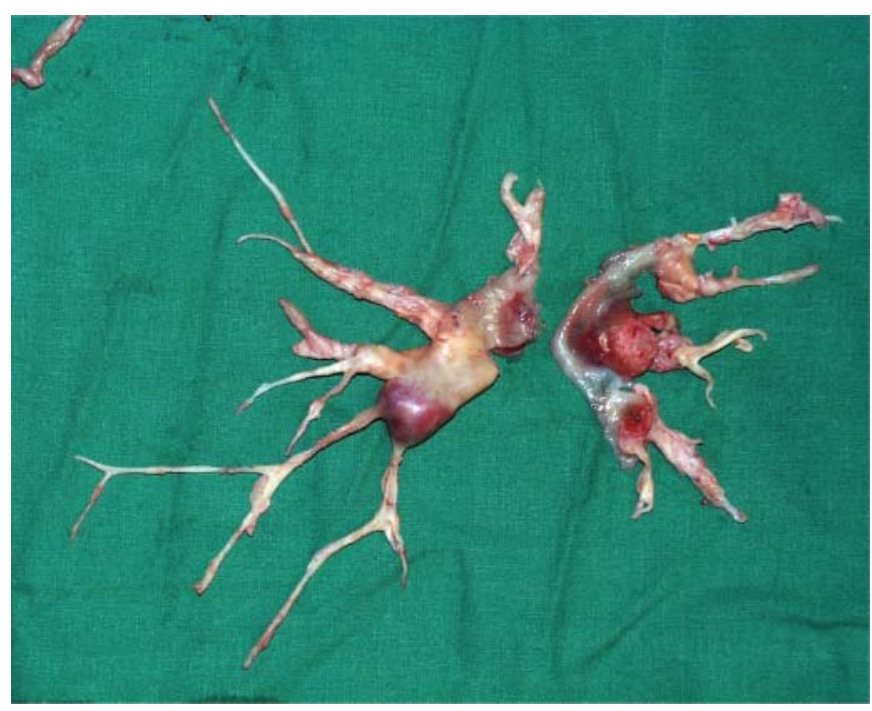

FIGURE 1. A pulmonary endarterectomy specimen. It is possible to perform endarterectomy down to segmental branches of pulmonary arteries. Reproduced from [14] with permission from the publisher. 
diuretics, inotropics, continuous venovenous haemofiltration and extracorporeal membrane oxygenation. Factors that are associated with death after PEA include residual pulmonary hypertension, right ventricular failure and lung reperfusion injury [5].

Post-operative treatment protocols vary even among expert centres. Selective pulmonary vasodilation with inhaled iloprost can be beneficial after surgery: in contrast with placebo, a single dose of iloprost administered immediately after surgery led to significant reductions in mean Ppa and PVR in a small, randomised study of patients with residual pulmonary hypertension after PEA [17]. Early reocclusion prophylaxis with heparin is mandatory after PEA, as is lifelong therapeutic anticoagulation (international normalised ratio target range 2.5-3.5), which should be started as soon as possible following surgery [1]. Patient-controlled anticoagulation therapy may improve outcome and reduce complications. No consensus exists on the potential benefits of pre-operative insertion of a vena-cava filter [1].

\section{OUTCOME}

To date, approximately 4,000 PEA operations have been performed worldwide. Results from specialised centres are excellent, with post-operative mortality rates ranging from $<5 \%$ to $\sim 10 \%$, depending on the level of experience of the surgical team [1, 4]. At the University of California, early mortality after PEA decreased from $9.1 \%$ for the first 500 patients to $6.7 \%$ for the following 1,000 patients (overall mortality $7.5 \%$ ) [15]. Our own outcomes from the Johannes Gutenberg University Hospital (Mainz, Germany) and Kerckhoff Heart and Lung Centre (Bad Nauheim, Germany) show similar trends of improved survival with increasing levels of surgical experience (table 2). Significant improvements in exercise capacity and haemodynamics are achieved in most patients after PEA, as are good long-term improvements in quality of life and life expectancy [1]. An improved postPEA peak oxygen uptake that was subsequently maintained at 1-2 yrs after surgery has also been reported [18]. The proportion of patients with persistent or recurrent pulmonary hypertension after PEA is believed to be between 10-20\% [7].

\section{MEDICAL TREATMENT OF INOPERABLE CTEPH}

Data from pulmonary hypertension centres in the UK suggest that, in recent years, the prognosis has also improved for patients with inoperable CTEPH, but that initial functional improvements do not persist far beyond the first year after

\begin{tabular}{|c|c|c|c|}
\hline TABLE 2 & \multicolumn{3}{|c|}{$\begin{array}{l}\text { Pulmonary endarterectomy team experience and } \\
\text { early mortality after pulmonary endarterectomy } \\
\text { between April } 1994 \text { and April } 2009 \text { in Mainz and } \\
\text { Bad Nauheim, Germany }\end{array}$} \\
\hline Study period & & Patients & Early mortality \\
\hline \multicolumn{2}{|c|}{ April 1994 to July 2003} & 210 & $23(10.9)$ \\
\hline \multicolumn{2}{|c|}{ August 2003 to April 2009} & 210 & $11(5.2)$ \\
\hline \multicolumn{2}{|c|}{ Overall ${ }^{\#}$} & 420 & $34(8.1)$ \\
\hline
\end{tabular}

diagnosis [8]. There are currently no approved medical therapies for CTEPH and no compelling data from randomised controlled trials that support the efficacy of approved pulmonary hypertension therapies in patients with CTEPH $[19,20]$. A randomised trial of bosentan in inoperable forms of CTEPH (i.e. the Bosentan Effects in iNopErable Forms of chronIc Thromboembolic pulmonary hypertension study) showed a $24 \%$ reduction in PVR, compared with placebo $(p<0.001)$, but did not reach its co-primary end-point of an improved 6-min walking distance $[19,21]$. In the current issue of the European Respiratory Review, a more in-depth discussion of pulmonary hypertension pharmacotherapies in CTEPH is provided by KIM [22].

\section{CONCLUSIONS}

Symptomatic and haemodynamic improvements following PEA are persistent in most patients with CTEPH. Supporting data for PEA from randomised, placebo-controlled trials are not available, as it would be unethical to withhold treatment in these severely ill patients. Notwithstanding, surgery is likely to remain the mainstay of therapy for patients with CTEPH. However, the effect, if any, of PEA on secondary vascular remodelling that was established before surgery remains to be elucidated.

\section{STATEMENT OF INTEREST}

E. Mayer has received speaker and consulting fees from Bayer Schering Pharma, Actelion and Pfizer.

\section{ACKNOWLEDGEMENTS}

Medical writing support was provided by A. Becher (Oxford PharmaGenesis Ltd, Oxford, UK) on behalf of Bayer Schering Pharma AG (Berlin, Germany). This article is based on a presentation given at a symposium supported by Bayer Schering Pharma AG at the 2009 European Society of Cardiology meeting in Barcelona, Spain.

\section{REFERENCES}

1 Keogh AM, Mayer E, Benza RL, et al. Interventional and surgical modalities of treatment in pulmonary hypertension. J Am Coll Cardiol 2009; 54: Suppl. 1, S67-S77.

2 Galie N, Kim NH. Pulmonary microvascular disease in chronic thromboembolic pulmonary hypertension. Proc Am Thorac Soc 2006; 3: 571-576.

3 Pepke-Zaba J. Diagnostic testing to guide the management of chronic thromboembolic pulmonary hypertension: state of the art. Eur Respir Rev 2010; 19: 55-58.

4 Mayer E. Surgical treatment of chronic thromboembolic pulmonary hypertension. Swiss Med Wkly 2006; 136: 491-497.

5 Fedullo PF, Auger WR, Kerr KM, et al. Chronic thromboembolic pulmonary hypertension. N Engl J Med 2001; 345: 1465-1472.

6 Dartevelle P, Fadel E, Mussot S, et al. Chronic thromboembolic pulmonary hypertension. Eur Respir J 2004; 23: 637-648.

7 Bonderman D, Skoro-Sajer N, Jakowitsch J, et al. Predictors of outcome in chronic thromboembolic pulmonary hypertension. Circulation 2007; 115: 2153-2158.

8 Condliffe R, Kiely DG, Gibbs JS, et al. Improved outcomes in medically and surgically treated chronic thromboembolic pulmonary hypertension. Am J Respir Crit Care Med 2008; 177: 1122-1127.

$9 \mathrm{Kim} \mathrm{NH}$. Assessment of operability in chronic thromboembolic pulmonary hypertension. Proc Am Thorac Soc 2006; 3: 584-588.

10 Thistlethwaite PA, Kaneko K, Madani MM, et al. Technique and outcomes of pulmonary endarterectomy surgery. Ann Thorac Cardiovasc Surg 2008; 14: 274-282. 
11 Condliffe R, Kiely DG, Gibbs JS, et al. Prognostic and aetiological factors in chronic thromboembolic pulmonary hypertension. Eur Respir J 2009; 33: 332-338.

12 Reesink HJ, Surie S, Kloek JJ, et al. Bosentan as a bridge to pulmonary endarterectomy for chronic thromboembolic pulmonary hypertension. J Thorac Cardiovasc Surg 2010; 139: 85-91.

13 Bresser P, Fedullo PF, Auger WR, et al. Continuous intravenous epoprostenol for chronic thromboembolic pulmonary hypertension. Eur Respir J 2004; 23: 595-600.

14 Hoeper MM, Mayer E, Simonneau G, et al. Chronic thromboembolic pulmonary hypertension. Circulation 2006; 113: 2011-2020.

15 Jamieson SW, Kapelanski DP, Sakakibara N, et al. Pulmonary endarterectomy: experience and lessons learned in 1,500 cases. Ann Thorac Surg 2003; 76: 1457-1462.

16 Mayer E, Klepetko W. Techniques and outcomes of pulmonary endarterectomy for chronic thromboembolic pulmonary hypertension. Proc Am Thorac Soc 2006; 3: 589-593.
17 Kramm T, Eberle B, Guth S, et al. Inhaled iloprost to control residual pulmonary hypertension following pulmonary endarterectomy. Eur J Cardiothorac Surg 2005; 28: 882-888.

18 Matsuda H, Ogino H, Minatoya K, et al. Long-term recovery of exercise ability after pulmonary endarterectomy for chronic thromboembolic pulmonary hypertension. Ann Thorac Surg 2006; 82: 1338-1343.

19 Lang IM. Managing chronic thromboembolic pulmonary hypertension: pharmacological treatment options. Eur Respir Rev 2009; 18:24-28.

20 Olschewski H. Inhaled iloprost for the treatment of pulmonary hypertension. Eur Respir Rev 2009; 18: 29-34.

21 Jais X, D'Armini AM, Jansa P, et al. Bosentan for treatment of inoperable chronic thromboembolic pulmonary hypertension: BENEFiT (Bosentan Effects in iNopErable Forms of chronIc Thromboembolic pulmonary hypertension), a randomized, placebo-controlled trial. J Am Coll Cardiol 2008; 52: 2127-2134.

22 Kim NH. Riociguat: an upcoming therapy in chronic thromboembolic pulmonary hypertension? Eur Respir Rev 2010; 19: 68-71. 